\title{
Access and use of health services by quilombolas with hypertension
}

\section{Acesso e utilização dos serviços de saúde por quilombolas com hipertensão}

\section{Acceso y uso de los servicios de salud por quilombolas con hipertensión}

\author{
Rosilene das Neves Pereira1 (iD), Ricardo Franklin de Freitas Mussi² iD, \\ Claudio Bispo de Almeida ${ }^{3}$ iD, Roseanne Montargil Rocha ${ }^{4}$ (i)
}

\footnotetext{
1 Universidade Estadual do Sudoeste da Bahia, Jequié, Bahia, Brasil.

2 Universidade do Estado da Bahia, Caetité, Bahia, Brasil.

3 Universidade do Estado da Bahia, Guanambi, Bahia, Brasil.

${ }^{4}$ Universidade Estadual de Santa Cruz, Ilhéus, Bahia, Brasil.
}

Autor correspondente:

Rosilene das Neves Pereira

E-mail: rose.pereira1987@hotmail.com

Como citar: Pereira, R. N., Mussi, R. F. F., Almeida, C. B., \& Rocha, R. M. (2021). Access and use of health services by quilombolas with hypertension. Journal of Research and Knowledge Spreading, 2(1), e12313.

http://dx.doi.org/10.20952/jrks2112313

\section{ABSTRACT}

The present study aimed to describe the access and use of health services by hypertensive individuals living in quilombola communities in Bahia. This is a population-based descriptive investigation, with data obtained through the application of a validated questionnaire for the quilombola population, $\geq 18$ years old, of both sexes. Hypertension was determined by selfreferral medical diagnosis. And, questions regarding access and use of the system and health were obtained. Blood pressure was measured by $72.7 \%$ of participants in the last six months. Hypertension was prevalent in $28.0 \%$ of the population, higher in the elderly and in women. The diagnoses occurred at $55.3 \pm 14.7$ years on average. Among the sick, $55.5 \%$ cited consultations to monitor their clinical status, while $49.7 \%$ purchased all medicines from public health services. There is a discontinuity of assistance or use of health services, so it seems a misconception the indication of underutilization of services by quilombolas, the problem is the absence and/or barriers to access and accessibility for appropriate use to demand.

Keywords: Access to Health Services; Epidemiological Survey; Black Population; Hypertension. 


\section{RESUMO}

O presente estudo teve o objetivo de descrever o acesso e a utilização dos serviços de saúde por hipertensos residentes em comunidades quilombolas de região baiana. Trata-se de uma investigação descritiva de base populacional, com obtenção de dados pela aplicação de questionário validado para população quilombola, com $\geq 18$ anos, de ambos os sexos. A hipertensão arterial foi determinada pela autorreferência de diagnóstico médico. E, foram obtidas questões referentes ao acesso e a utilização dos sistema e saúde. A pressão arterial foi aferida por $72,7 \%$ dos participantes nos últimos seis meses. A hipertensão foi prevalente em $28,0 \%$ da população, maior em idosos e nas mulheres. Os diagnósticos ocorreram aos 55,3 14,7 anos em média. Entre os enfermos, 55,5\% citaram realização de consultas para acompanhamento do quadro clínico, enquanto $49,7 \%$ adquiriram todos os medicamentos nos serviços públicos de saúde. Existe uma descontinuidade da assistência ou do uso dos serviços de saúde, dessa maneira, parece um equívoco a indicação de subutilização dos serviços pelos quilombolas, a problemática são as ausências e/ou às barreiras para o acesso e acessibilidade para utilização adequada à demanda.

Palavras-chave: Acesso aos Serviços de Saúde; Inquérito Epidemiológico; População Negra; Hipertensão.

\section{RESUMEN}

El presente estudio tuvo como objetivo describir el acceso y uso de los servicios de salud por personas hipertensas que viven en comunidades quilombolas en Bahía. Se trata de una investigación descriptiva de base poblacional, con datos obtenidos mediante la aplicación de un cuestionario validado para la población quilombola, $\geq 18$ años, de ambos sexos. La hipertensión arterial se determinó mediante un diagnóstico médico autonotificado. Y se obtuvieron preguntas sobre acceso y uso del sistema y salud. El 72,7\% de los participantes midió la presión arterial en los últimos seis meses. La hipertensión fue prevalente en el 28,0\% de la población, mayor en ancianos y mujeres. Los diagnósticos se produjeron a los 55,3+14,7 años en promedio. Entre los enfermos, el 55,5\% citó consultas para vigilar su estado clínico, mientras que el 49,7\% adquirió todos los medicamentos de los servicios de salud pública. Existe una discontinuidad de la asistencia o el uso de los servicios de salud, por lo que parece un error indicar una subutilización de los servicios por parte de los quilombolas, el problema son las ausencias y/o barreras de acceso y accesibilidad para un uso adecuado a la demanda.

Palabras clave: Acceso a los servicios de salud. Encuesta epidemiológica. Población negra. Hipertensión.

\section{INTRODUÇÃO}

A utilização dos serviços de saúde pode ser descrita como os fatores relacionados à necessidade de saúde da população referente à morbidade, gravidade e urgência da doença, ou ainda aspectos organizacionais que incluem recursos disponíveis e características da oferta como disponibilidade de profissionais de saúde, unidades de atendimento entre outras características que interagem com questões demográficas, geográficas, socioeconômicas, culturais, psíquicas, entre outras (Travassos \& Martins, 2004).

Nessa perspectiva, existem vários fatores que podem dificultar o processo de acesso e utilização dos serviços de saúde e no contexto das pessoas residentes em comunidades quilombolas rurais as barreiras podem ser de 'acessibilidades econômicas, organizacionais e culturais que se interpõem entre a oferta de serviços e o atendimento efetivo e oportuno das necessidades dessas populações (Castellano \& Guimarães, 2012; Pereira et. al., 2020; Pereira \& Mussi, 2020). Em estudo de reflexão, considerou-se que as relações de poder existentes nos 
sistemas de saúde brasileiro pode ser um dos aspectos que dificultam o acesso dos quilombolas à saúde pública, marcado historicamente por desigualdades de direitos sociais, o que privilegia aqueles que detem o poder político (Almeida et. al., 2019). As relações de poder que levaram a população negra brasileira à condições sociais desfavoráveis, que tratam esta população como "colonizado" seja no ambiente escolar (Borja; Pereira, 2018) no sistema de saúde (Almeida et. al., 2019), por meio da imposição de um racismo estruturado na sociedade em geral. Tal situação mostra a necessidade de, cada vez mais, a população negra buscar exercer o poder na sociedade (Santos \& Lucini, 2021), sobretudo, as populações quilombolas.

Partindo desse pressuposto, reconhece-se a relevância em realizar estudos que analisem a perspectiva da utilização dos serviços de saúde pela população quilombola com hipertensão, pois algumas pesquisas tem evidenciado um elevado índice de Hipertensão Arterial Sistêmica (HAS) em indivíduos quilombolas rurais (Bezerra et. al., 2013; Bezerra et. al., 2015; Melo \& Silva, 2015; Silva \& Menezes, 2016; Belfort et. al., 2017; Matos et. al., 2021).

Estima-se que a prevalência global da HAS tenha ultrapassado um bilhão de casos, acarretando aproximadamente 7,1 milhões de mortes ao ano (Brasil, 2012). 0 primeiro inquérito representativo da população brasileira identificou HAS prevalente em 21,4\% dos adultos, mais recorrente em mulheres e entre indivíduos a partir dos 75 anos de idade (Andrade et. al., 2013), sendo esse um dado preocupante, pois, a HAS é o principal fator de risco para as doenças isquêmicas do coração, doenças cerebrovasculares e também a principal causa de óbito prevenível no mundo, responsável por 13\% das mortes (Who, 2011). E, é importante considerar que a HAS faz parte dos componentes de outra condição que pode agravar a saúde desta população, como por exemplo, a síndrome metabólica, que em estudo realizado nesta população apresentou prevalência de 25,8\% (Mussi \& Petroski, 2019).

Nesse contexto, verifica-se a necessidade de um amplo acesso aos serviços de saúde para prevenção, diagnóstico precoce e orientações para o manejo adequado da doença (Bezerra et. al., 2013). Assim, julga-se pertinente descrever o acesso e a utilização dos serviços de saúde por hipertensos residentes em comunidades quilombolas de região baiana.

\section{METODOLOGIA}

Esta análise trata de um estudo que utiliza dados da pesquisa transversal de base populacional intitulada "Perfil Epidemiológico dos Quilombolas baianos", autorizada pelo Comitê de Ética em Pesquisa com Seres Humanos da Universidade do Estado da Bahia, sob o parecer $\mathrm{n}$ ㅇ 1.386.019/2016, com coletas realizadas entre fevereiro e novembro de 2016 .

A microrregião geográfica de Guanambi/Bahia é composta por 18 municípios e possui área territorial de 22.668,688 quilômetros quadrados, com 42 quilombos contemporâneos. Destas comunidades, 40 eram certificadas até o ano de 2016 (Fundação Palmares, 2016) distribuídas em 10 municípios, e representaram o campo empírico investigado. Diante da indisponibilidade de informações oficiais prévias relativas à quantidade de moradores dos quilombos desta microrregião baiana, a população foi estimada considerando 80 famílias por quilombo (Secretaria de Políticas de Promoção da Igualdade Racial da Presidência da República, 2010), com dois adultos (>18 anos) por família, totalizando 6720 adultos.

O cálculo amostral adotou correção para população finita, prevalência para desfecho desconhecido (50\%), confiança de 95\%, erro amostral de cinco pontos percentuais, correção de 1,5 vezes para conglomerado em um estágio (quilombo), acréscimos de $30 \%$ para recusas e $20 \%$ para perdas e confundimento, que determinou amostra mínima de 818 sujeitos.

0 desenho amostral consistiu em duas etapas: sorteio dos quilombos (conglomerado) e, em seguida, coleta censitária. Inicialmente foi realizado sorteio aleatório dos quilombos, que por meio das respectivas associações de moradores (órgãos locais que representam os interesses dos quilombolas) poderiam permitir ou não as visitações para a realização da pesquisa. 
Todos os adultos (idade igual o maior que 18 anos) residentes nos quilombos participantes durante o período da coleta foram considerados elegíveis, sendo convidados e informados sobre os aspectos do estudo, garantindo igual probabilidade de participação.

Para obtenção das informações referente à existência de unidade de saúde bem como de membro da Equipe de Saúde da Família nos quilombos, realizou-se entrevista estruturada a um integrante de cada Associação de Moradores das Comunidades Quilombolas, com perguntas relativas à presença de infraestrutura física e/ou serviços públicos de saúde e presença de Agente Comunitário de Saúde (ACS) lotados nas 14 respectivas áreas.

Quanto à investigação das condições de saúde da população, foram realizadas coletas em sistema de mutirão, durante os finais de semana e feriados. Dessa forma, 1025 quilombolas compareceram as atividades, dos quais 850 aceitaram participar voluntariamente, por meio da assinatura ou fornecimento da impressão digital no Termo de Consentimento Livre e Esclarecido individual, compondo a amostra final. Os convidados que não compareceram nas atividades caracterizaram as recusas. A identificação de dado faltante durante a análise foi considera perda informacional.

A coleta de dados sobre as condições de saúde foi realizada por meio de entrevistas. Estas atividades foram desenvolvidas por equipes compostas por profissionais e/ou acadêmicos da área de saúde conforme suas habilitações, após treinamento para sua respectiva função. Quanto aos participantes da pesquisa, aqueles com deficiência cognitiva ou de comunicação independente, foram excluídos por não compor os critérios de elegibilidade para esse estudo.

Inicialmente para a análise considerou-se o percentual de pessoas que informaram ter mensurado a pressão pela última vez (categorizado em: $<6$ meses; 6 meses- $<1$ ano; 1 ano- $<2$ anos; 2-anos-<3 anos; $>3$ anos e nunca);

- Percentual de pessoas com diagnóstico médico autorreferido para HAS (categorias dicotômicas sim ou não);

Para análise das variáveis sociodemográficas consideraram-se: sexo (masculino e feminino); grupo etário (adulto e idoso); raça/cor (negro e outros); escolaridade (< de 5 anos e $>5$ anos) e renda Familiar (conforme salário mínimo vigente no período, $\mathrm{R} \$<880,00$ e $>880,00$ ). Quanto a prevalência, esta foi calculada segundo sexo e grupo etário e a média foi calculada para idade e renda familiar.

Com relação a descrição das variáveis sobre uso do serviço para acompanhamento da HAS consideraram-se:

- Percentual de pessoas que indicaram realização de consultas regulares nos serviços de saúde por causa da HAS (categorizado em: sim; quando tem algum problema; nunca);

- Percentual de pessoas que informaram utilização de medicamentos nas duas últimas semanas por causa da HAS (categorias dicotômicas sim ou não);

- Percentual de pessoas que citaram obtenção de medicamentos para HAS em serviço público de saúde (categorizado em: todos; alguns; nenhum);

- Percentual de pessoas afirmaram ter recebido, na última consulta, assistência médica por causa da HAS (categorizado em: $<6$ meses; 6 meses- $<1$ ano; 1 ano- $<2$ anos; 2 -anos$<3$ anos; $>3$ anos e nunca);

- Percentual de pessoas que comunicaram atendimento pelo SUS (categorias dicotômicas: sim ou não);

- Percentual de pessoas que tiveram internação por causa da HAS ou de alguma complicação (categorias dicotômicas: sim ou não);

- Percentual de pessoas que executaram atividades habituais com graus de limitação devido a HAS ou alguma complicação da hipertensão (não; pouco; moderada; intensa; muito);

- Recomendações recebidas de algum médico ou outro profissional de saúde quanto a alimentação saudável; manter o peso adequado; ingerir menos sal; praticar atividade 
física regular; não beber álcool em excesso e fazer o acompanhamento regular da HAS (respostas em categorias dicotômicas: sim ou não).

\section{RESULTADOS}

Em um total de 17 comunidades quilombolas contatadas após o sorteio, 14 permitiram o desenvolvimento do estudo. Participaram deste estudo 850 quilombolas, mesmo após as recusas individuais, que totalizaram 17,07\%. A amostra final para o desfecho HAS foi constituída por 825 participantes.

Foi identificado que $72,7 \%$ dos entrevistados referiram participação em atendimento que conteve aferição da pressão arterial (PA) nos últimos 6 meses. Destes, 28,0\% indicaram possuir o diagnóstico médico de HAS (Tabela 1).

Dos participantes que referiram possuir HAS: 63,9\% eram adultos, 68,9\% do sexo feminino, 85,0\% negros, 80,5\% com escolarização até a 4 a série, e 73,6\% tinham renda familiar de até R \$ 880,00(salário mínimo no período). A prevalência da doença foi de $56,1 \%$ em idosos, $32,8 \%$ em mulheres e a média de idade para o diagnóstico foi de $55,32 \pm 14,73$ anos e a renda foi de $807,63 \pm 603,23$ reais.

Verificou-se que dos adultos com HAS, 55,5\% realizam consultas regulares, $77,2 \%$ utilizaram medicações nas duas últimas semanas, com 49,7\% citando sua aquisição exclusivamente em serviços públicos de saúde. Sobre assistência médica por causa da HAS, $59,7 \%$ dos hipertensos efetuaram consultas em menos seis meses, 9,7\% em período maior que três anos e 8,4\% informaram que nunca realizaram. Sobre o local de atendimento 91,4\% dos entrevistados indicaram terem sido atendidos nos serviços públicos. No que tange às complicações por causa da enfermidade, 29,2\% referiram internação e 38,3\% informaram algum grau de limitação para desenvolvimento das atividades habituais (Tabela 1).

Tabela 1- Utilização dos serviços de Saúde pelos quilombolas com diagnóstico médico autorreferido para Hipertensão, Bahia, Brasil, 2016

\begin{tabular}{|c|c|}
\hline Quando foi a última vez que o(a) Sr.(a) teve sua pressão arterial medida? $(\mathrm{n}=825)$ & $\%$ (n) \\
\hline$<6$ meses & $72,7 \%(600)$ \\
\hline 6 meses-<1ano & $13,3 \%(110)$ \\
\hline 1 ano- $<2$ anos & $5,7 \%(47)$ \\
\hline $2-<3 a n o s$ & $3,1 \%(26)$ \\
\hline$>$ 3anos & $3,2 \%(27)$ \\
\hline Nunca & $1,8 \%(15)$ \\
\hline \multicolumn{2}{|l|}{$\begin{array}{l}\text { Algum médico já lhe deu o diagnóstico de hipertensão arterial (pressão alta)? } \\
(n=826)\end{array}$} \\
\hline $\operatorname{Sim}$ & $28,0 \%(231)$ \\
\hline Gravidez & $1,2 \%(10)$ \\
\hline Não & $70,8 \%(585)$ \\
\hline \multicolumn{2}{|l|}{$\begin{array}{l}\text { Quando foi a última vez que o(a) Sr.(a) recebeu assistência médica por causa da } \\
\text { hipertensão arterial? }(n=226)\end{array}$} \\
\hline$<6$ meses & $59,7 \%(135)$ \\
\hline 6 meses-<1ano & $8,8 \%(20)$ \\
\hline 1 ano- $<2$ anos & $8,8 \%(20)$ \\
\hline $2-<3 a n o s$ & $4,4 \%(10)$ \\
\hline$>$ 3anos & $9,7 \%(22)$ \\
\hline Nunca & $8,4 \%(19)$ \\
\hline \multicolumn{2}{|l|}{ Esse atendimento foi feito pelo SUS? $(n=210)$} \\
\hline Sim & $91,4 \%(192$ \\
\hline Não & $8,6 \%(18)$ \\
\hline
\end{tabular}




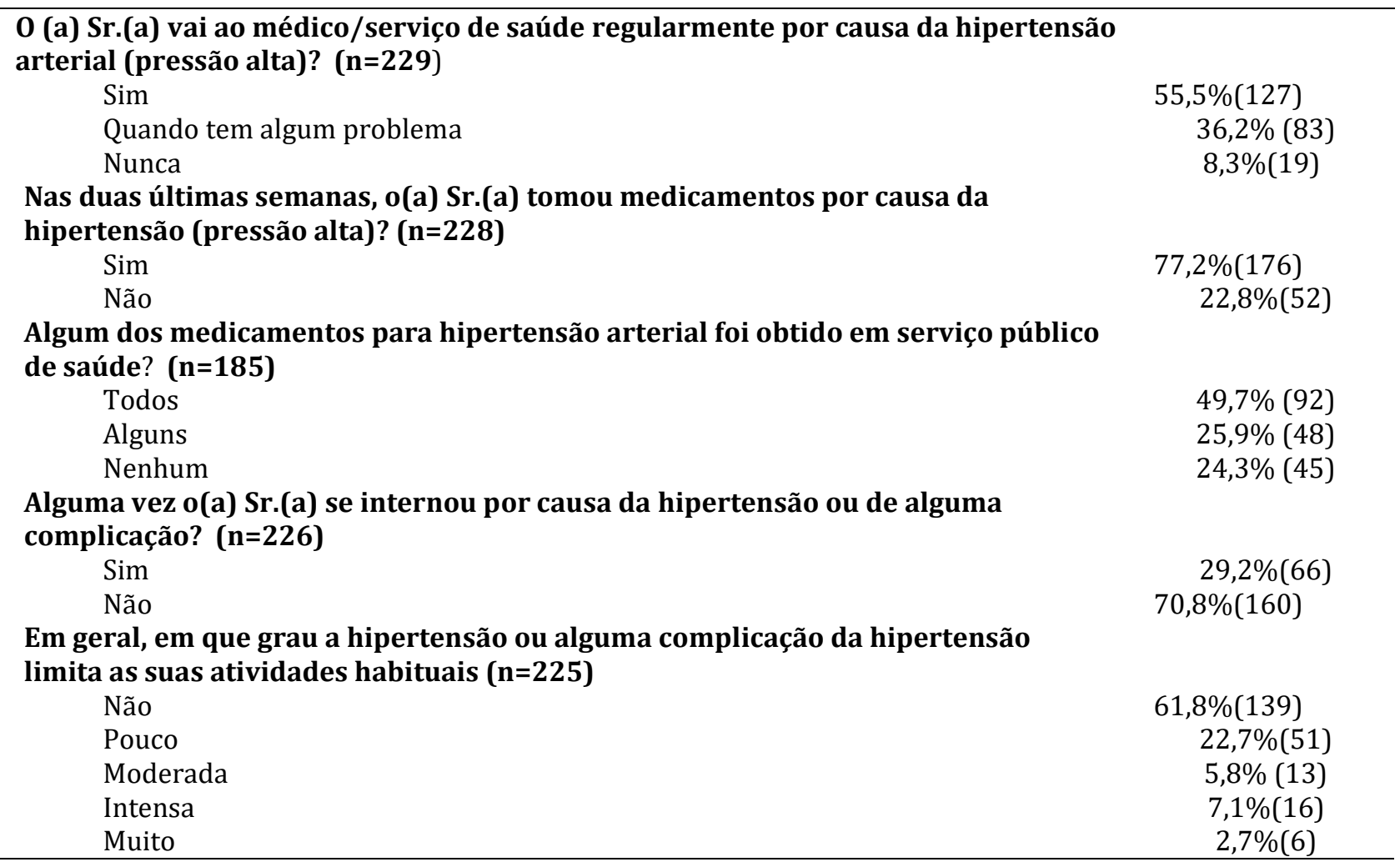

Quanto às recomendações dos profissionais de saúde, 78,0\% dos hipertensos informaram ter recebido orientação sobre a importância de manter a alimentação saudável, $61,4 \%$ afirmaram ter recebido orientação para manter o peso adequado, 81,2\% para ingerir menos sal, 59,2\% para praticar atividade física regularmente, 55,6\% receberam orientação para não ingerir bebidas alcoólicas em excesso e 69,5\% para acompanhamento da enfermidade de maneira regular (tabela 2).

Tabela 2- Recomendações dos profissionais de saúde para os adultos quilombolas durante as consultas de HAS, Bahia, Brasil, 2016

\begin{tabular}{lc}
\hline $\begin{array}{l}\text { Orientação recebida de algum médico ou outro profissional de saúde para } \\
\text { manter uma alimentação saudável? (n=223) }\end{array}$ & \% (n) \\
$\quad$ Sim \\
$\begin{array}{l}\text { Orientação recebida de algum médico ou outro profissional de saúde para } \\
\text { manter o peso adequado? (n=223) }\end{array}$ & $78,0 \%(174)$ \\
$\quad \begin{array}{l}\text { Sim } \\
\text { Orientação recebida de algum médico ou outro profissional de saúde para } \\
\text { ingerir menos sal? (n=223) }\end{array}$ & $61,4 \%(137)$ \\
$\quad \begin{array}{l}\text { Sim } \\
\text { Orientação recebida de algum médico ou outro profissional de saúde para } \\
\text { praticar atividade física regular? (n=223) }\end{array}$ & $81,2 \%(181)$ \\
$\quad \begin{array}{l}\text { Sim } \\
\text { Orientação recebida de algum médico ou outro profissional de saúde para não } \\
\text { beber em excesso? (n=223) }\end{array}$ & $59,2 \%(132)$ \\
$\quad$ Sim \\
$\begin{array}{l}\text { Orientação recebida de algum médico ou outro profissional de saúde para fazer } \\
\text { o acompanhamento regular da HAS? (n=223) } \\
\quad \text { Sim }\end{array}$ \\
\hline
\end{tabular}


0 estudo revelou que dos 825 entrevistados, 72,7\% informaram ter aferido a pressão pela última vez em período menor que 6 meses e 28,0\% indicaram ter diagnóstico médico de HAS. Pondera-se que o quantitativo de pessoas que relataram possuir diagnóstico médico de hipertensão não pode ser considerado como o real percentual de pessoas hipertensas no cenário estudado, uma vez que as barreiras interpostas no acesso aos serviços contribuem imensamente para o subdiagnóstico da doença.

Sobre a subestimação do número de pessoas com diagnóstico de HAS (Bezerra et. al., 2015), estudo identificou que quase metade dos quilombolas hipertensos desconheciam o diagnóstico da doença e o fato do indivíduo saber que possui hipertensão não se constitui por si só como garantia para o controle da PA, apesar do conhecimento ser considerado um passo primordial.

As desigualdades sociais, geralmente vivenciadas por populações afrodescendentes em relação a outras populações têm justificado as dificuldades de acesso ao diagnóstico e tratamento da HAS bem como contribuído para sua alta prevalência (Kurian; Cardarelli, 2007). Além de distantes geograficamente, quando os equipamentos/serviços de saúde estão presentes nos territórios quilombolas, caracterizam-se pela limitada inserção, aceitabilidade e diálogo com os práticas e saberes locais ${ }^{14}$. Outra forma de desigualdade pode ser observada no não cumprimento dos direitos à saúde adquirido por esta população, que reflete no sistema de saúde brasileiro, que não consegue garantir um acesso equânime (Almeida et. Al., 2019).

Essa condição permite afirmar que a discrepância entre as oportunidades dispensadas às populações na perspectiva do acesso a serviços e bens, impacta diretamente nas formas de cuidado com a saúde, e na promoção e prevenção da saúde, pois, as comunidades quilombolas são de fato carentes de serviços como unidades de saúde da família, academia ao ar livre, grupos de atividades físicas, Núcleo de Apoio à Saúde da Família (NASF), entre outros.

A adequação da infraestrutura e do funcionamento do serviço de saúde local, bem como a realização de campanhas de promoção da saúde, pode contribuir para a prevenção, diagnóstico precoce e tratamento da hipertensão e outros agravos (Silva; Menezes, 2016). Entretanto, torna-se pertinente pontuar que a oferta do serviço e implementação de infraestrutura não são suficientes o bastante para garantir que os quilombolas tenham acesso a uma assistência de qualidade devido ao racismo institucional, questões socioculturais e as relações profissional-paciente ainda permeada por inadequações, fruto do racismo institucional.

No entanto, não basta ampliar a rede de atenção básica nos espaços quilombolas. É necessário que os serviços se dediquem a harmonização da prática técnico-científicos com realidade das populações negras (Paranhos; Cardoso, 2021), enfocando a equidade como princípio fundamental ao cumprimento das diretrizes do SUS e potencializando o acesso integral aos serviços para o enfrentamento das desigualdades presentes nestes territórios (Fernandes; Santos, 2019).

Evitar a ação verticalizada na execução das ações de saúde é fundamental para enfrentamento do racismo imbricado na maneira de pensar e de implementar políticas, que influenciam no processo de lotação de estabelecimentos de saúde, regularmente construídos distantes dos quilombos, desrespeitando suas demandas geopolíticas especificas. Diante dessa conjuntura da organização pautada na urbanização, que prioriza localidades com maior densidade demográfica, é preciso ressaltar que não são as comunidades quilombolas que se localizam distantes das unidades de saúde e sim os estabelecimentos de saúde que são construídos sem analisar quem mais tem carência dos serviços públicos. E isso, por sua vez, contribui consideravelmente para uma busca do serviço pautada nas situações emergenciais gerando uma descontinuidade da procura bem como da assistência e consequentemente do (des)acompanhamento da hipertensão. 
A esse respeito a descontinuidade do cuidado ou tratamento da hipertensão, se deve a questões como localização geográfica estritamente rural (Travassos; Viacava, 2007).Porém, também deve ser considerado o impacto do racismo na geração/reforço de condições socioeconômicas vulnerabilizantes da população quilombola, que contribuem para o menor controle dos níveis pressóricos (Bezerra et. al., 2014). Pois, ainda que a geografia se constitua como importante barreira para o acesso e utilização do serviço, baixos níveis econômicos e de escolaridade, dificultam a adesão dos quilombolas ao tratamento adequado da HAS.

Sobre o perfil dos hipertensos, o estudo identificou prevalência de 56,1\% em idosos e $32,8 \%$ em mulheres e a idade média do diagnóstico foi de $55,3 \pm 14,7$ anos para os hipertensos

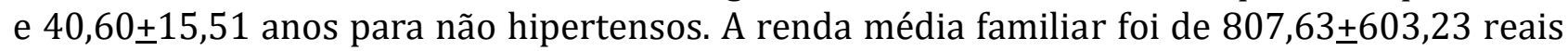
para hipertensos e não hipertensos $673,14 \pm 547,29$, corroborando com o encontrado em outras investigações com quilombolas (Bezerra et. al., 2015; Silva; Menezes, 2016; Bezerra et. el., 2014).

A prevalência de HAS entre as mulheres corroborou com a literatura (Silva; Menezes, 2016; Ferreira, et al., 2006; Lessa et. al., 2006). Esse resultado pode ser explicado devido as mulheres possuírem maior autocuidado e buscarem mais assistência médica do que os homens, algo que contribui para elas apresentarem maiores percentuais de reconhecimento da doença (Nogueira et. al., 2010).

Corroborando com os resultados da presente investigação, a Pesquisa Nacional de Saúde (Andrade et. Al., 2015) e estudos com quilombolas identificaram a idade associada à hipertensão (Bezerra et. al., 2013; Silva; Menezes, 2016). Nesse caso, sabe-se que o avançar da idade contribui para a progressão da HAS devido as alterações fisiológicas em vasos como, por exemplo, aorta, o que pode justificar a maior prevalência de HAS encontrada em pessoas com mais idade (Silva; Menezes, 2016; Miranda et. al., 2002).

A escolaridade e a renda per capita também demonstraram associação estatisticamente significativa com a HAS, com maiores prevalências entre os indivíduos de menor escolaridade e maior renda (Bezerra et. al., 2013; Silva; Menezes, 2016). A prevalência de HAS em quem possui maior renda foi um achado que divergiu do identificado em outros estudos (Ferreira et. al., 2006; Rosário et. al., 2009) o que pode ser justificado pelo extrato de renda, pois, o valor considerado como maior salário nas famílias quilombolas foi considerado como salário mínimo nas demais investigações (Nogueira et. al., 2010; Silva; Menezes, 2016).

Quanto ao uso regular do serviço para realização das consultas de hipertensão, apenas $55,5 \%$ dos entrevistados afirmaram ter realizado consultas regulares e 59,7\% indicaram ter efetuado consultas médicas em períodos menores que seis meses. Esse achado chamou atenção para duas situações importantes, a primeira foi sobre o percentual de pessoas $(44,5 \%)$ que tinham o diagnóstico, mas se encontravam sem o atendimento satisfatório (regular) para a doença, e a segunda situação refere ao entendimento do que seria considerado como consulta regular para a hipertensão, pois, normalmente evidencia-se uma procura pela consulta médica em menos de 6 meses pautada na troca de receita ou prescrição de medicamentos.

Neste sentido, investigação com enfoque nos serviço de controle de hipertensão e diabetes na atenção básica apontou que a formalização de políticas governamentais, como o Plano de Reorganização da Atenção à Hipertensão Arterial Sistêmica e ao Diabetes Mellitus, não tem refletido na melhoria dos indicadores de saúde na proporção almejada (Assis; Simões; Cavalcanti, 2012 $)^{22}$. Outros autores ainda ressaltam que é preciso pensar em iniciativas que melhore esse serviço e aponta como algumas alternativas o melhoramento do acompanhamento de usuários diagnosticados, a ampliação do acervo e da quantidade de medicamentos, a descentralização dos pontos de coleta de exames, sobretudo na zona rural, para facilitar o acesso a tais serviços bem como a intensificação do rastreamento de doentes (Carvalho Filha et. al., 2014).

De modo geral, verifica-se a necessidade de melhoria do serviço, pois, um estudo realizado em São Paulo mostrou um descontrole de diabetes e hipertensão com alto risco 
cardiovascular nos indivíduos pesquisados (Gerab et. al., 2012). Outro estudo desenvolvido no Rio de Janeiro apontou que apenas 44,9\% dos hipertensos apresentaram níveis de PA dentro da meta de níveis $<140 / 90 \mathrm{mmHg}$, assim, mostrando que há um longo caminho a ser percorrido e que o melhor investimento para melhoria deste parâmetro seria possivelmente a educação permanente dos médicos e demais profissionais envolvidos na assistência ao hipertenso (Novello et. al., 2017).

No cenário quilombola essa falta de controle da PA é persistente e autores afirmam ter observado maiores valores médios de PAS entre hipertensos quilombolas que conheciam o diagnóstico do que entre os hipertensos que desconheciam, revelando assim uma verdadeira descontinuidade do tratamento e descontrole dos níveis pressóricos por aqueles que possuem o diagnóstico (Bezerra et. al., 2015) ${ }^{4}$. Nesse sentido, verifica-se que a HAS é destacada como um problema de saúde pública na população quilombola, descortinando grande vulnerabilidade em saúde devido aos níveis insatisfatórios de conhecimento, controle e tratamento da doença.

Quanto o uso de medicamentos para controle da HAS nas duas últimas semanas anteriores a entrevista, 22,8\% (52) indicaram não ter feito uso. Sobre essa questão, um estudo realizado no Brasil mostrou que cerca de $17 \%$ dos indivíduos que referiram diagnóstico de HAS, não utilizavam medicamentos de uso contínuo e na análise univariada a frequência de indivíduos que não utilizavam os remédios regularmente foi maior entre os homens, os com escolarização de 8 a 10 anos de estudo, que referiram cor da pele/raça preta, pardos/indígenas, que viviam nas regiões rurais e nas macrorregiões Centro-oeste, Nordeste e Norte do país (Ferreira et. al., 2014).

Essa informação reforça o quanto as questões sociodemográficas contribuem para o uso irregular dos anti-hipertensivos pelos quilombolas. Estudos anteriores indicaram a distância das unidades de saúde como uma importante problemática, bem como, a inexistência ou precariedade do transporte público, que dificulta o acesso quilombola aos bens de saúde (Melo; Silva, 2015; Bezerra et. al, 2014).

Ainda no contexto da disponibilidade, foi investigada a aquisição de medicamentos, constatando-se que $49,7 \%$ dos hipertensos obtiveram todos os remédios para HAS nos serviços públicos de saúde, sendo esse um achado preocupante, pois, menos da metade dos hipertensos conseguiram obter todos os medicamentos nos serviços públicos e outra parcela $(50,2 \%)$ deixou de obter ou recorreu a serviços particulares para adquirir o restante das medicações ou se não todas. Isso, por sua vez, gera um problema/impasse para o usuário, pois, muitos deixarão de comprar o anti-hipertensivo prescrito por não possuir condições econômicas favoráveis para tal.

Nesse sentido, sabe-se que o custo do medicamento é importante para a adesão ao tratamento da HAS (Munge et. al., 2007) e a renda parece influenciar mais negativamente o acesso aos serviços do que a escolaridade (Travassos et. al., 2006). Desse modo, ter um serviço que disponibiliza todos os medicamentos e permite o acesso do usuário às consultas regulares, o que é extremamente importante.

Mediante essa realidade a farmácia popular seria uma oportunidade para os quilombolas terem acesso ampliado a essas medicações, pois a mesma funcionava com o propósito de levar o benefício da aquisição de medicamentos e insumos essenciais a baixo custo ou gratuitamente a mais lugares e mais pessoas, principalmente aquelas de baixa renda, haja visto que a falta de recurso financeiro aparece como sendo a principal causa de não uso dos medicamentos necessários para o tratamento da hipertensão (Gontijo et. al., 2012).

Os usuários do SUS têm como fonte primária de medicamentos o próprio SUS, entretanto há uma participação relevante de outras fontes de obtenção, indicando problemas no acesso dentro do SUS. No Sul e Sudeste, a população busca menos a farmácia privada do que no Nordeste e Norte do país (Matta et. Al., 2018).

Destarte, o SUS é apontado como um sistema fundamental para garantia do direito ao acesso universal e equânime à saúde, mas ainda é notável persistência de iniquidades nesse 
acesso. Desse modo, políticas públicas de combate à desigualdade social e de saúde devem ser implementadas para garantir a equidade nas comunidades quilombolas que, por sua vez são grupos vulneráveis (Bezerra et. al., 2014; Silva et. al., 2010; Barroso et. al., 2014; Kochergin et. al., 2014; Pereira; Mussi; Rocha, 2020; Pereira; Mussi, 2020) invisibilizados até hoje pelos gestores e sociedade em geral.

A garantia e ampliação do acesso e da efetividade dos serviços de saúde dependem da disponibilidade de recursos, e, considerando o papel exercido pelo SUS na atenção à saúde, torna-se salutar a busca por melhoria do financiamento da saúde pública (Viacava et. al., 2018) para que a assistência seja contemplativa.

No que tange às complicações da HAS, evidenciou que 29,2\% (66) dos quilombolas foram internados por causa da doença e 38, 2\% referiram apresentar algum grau de limitação em suas atividades habituais, fato que alerta os profissionais para empreenderem em sua rotina de trabalho momentos de orientações sobre os agravos causados pela HAS, uma vez que nesse estágio de cronicidade a doença poderá incapacitar o indivíduo devido repercussões como acidente vascular cerebral, infarto agudo do miocardio, Doença Renal Crônica entre outros complicações.

A assistência prestada através do serviço de controle de hipertensão e diabetes não se resume em aferição da Pressão Arterial (PA), mensuração dos níveis glicêmicos e/ou distribuição de fármacos, mas também, é um serviço que vem auxiliar na prevenção e redução dos agravos associados à hipertensão e diabetes que por sua vez tem como principal complicação a doença renal crônica e outros agravos cardiovasculares (Pereira et. al., 2018). Nesse sentido, torna-se extremamente relevante ressaltar que o percentual de quilombolas internados por causa da HAS impacta consideravelmente os cofres públicos, além disso, esses hipertensos são também aqueles grupos potenciais para incapacitação e/ou limitação nas atividades de vida diária.

A HAS pode gerar limitações importantes às pessoas, dessa maneira, o seu controle bem como o uso contínuo de um ou mais tipos de medicamentos anti-hipertensivos e mudanças no estilo de vida é considerado o tratamento adequado da doença e, portanto, medida fundamental para a redução da morbidade e mortalidade por doenças cardiovasculares (Chobanian et. al., 2003). Mas no contexto do público estudado (população negra) é possível considerar que o precário acesso as medicações podem dificultar o controle satisfatório da PA e que mais estudos precisam ser desenvolvidos para superar essa lacuna.

No que refere ao controle dos níveis pressóricos com participação de medidas nãofarmacológicas, é possível que o indivíduo que realize consultas médicas regulares, exames complementares e siga as recomendações quanto às modificações no estilo de vida, tais como: praticar atividade física, controlar o peso, ingerir menos sal, evitando o consumo de bebida alcoólica e alimentos gordurosos, além de não fumar (Sturmer et. al., 2006; SBH, 2010), possa adiar a inserção terapêutica dos medicamentos para quando esgotadas as demais alternativas (Veronez; Simões, 2008). No entanto, é importante ressaltar que o profissional médico é o responsável pela determinação do encaminhamento terapêutico farmacológico.

Estudo desenvolvido com quilombolas (Bezerra et. al., 2015) evidenciou que a maioria dos quilombolas receberam as recomendações sobre a modificação no estilo de vida, corroborando com os resultados encontrados nessa pesquisa. No entanto, é preciso questionar sobre como tem ocorrido essa orientação, pois é necessário que exista uma dinâmica dialógica entre profissionais e pacientes tornando o cuidado dotado de corresponsabilidade (Pereira et. al., 2018) e para que atenção à saúde seja realizada de forma adequada, deve haver também Equipes da Atenção Básica completas e em quantidade suficiente para atender à demanda existente, conforme o Ministério da Saúde recomenda e além disso, é necessário o apoio de outros profissionais de saúde como nutricionista, educador físico e farmacêutico (Borges; Lacerda, 2018). 
Sobre as limitações, o estudo evidenciou que a frequência de consulta médica dos quilombolas hipertensos foi em períodos menor que seis meses, no entanto, devido a não constar no questionário já previamente validado pela PNS (para utilização em quilombolas brasileiros >18 anos), não foi possível compreender sobre como ocorre essa procura para os demais profissionais como, por exemplo, o enfermeiro. Ademais a pesquisa teve limitação quanto ao delineamento transversal, desse modo, não sendo possível estabelecer relações de causa e efeito.

\section{CONCLUSÃo}

A utilização dos serviços de saúde pelos quilombolas hipertensos se dá de maneira bastante irregular devido a descontinuidade da assistência ou participação infrequente nas consultas. 0 levantamento demonstrou que três em cada 10 quilombolas apresentaram diagnóstico de HAS com prevalência de 56,1\% em idosos e 32,8\% em mulheres.

Esses achados são importantes para evidenciar a necessidade de discussões acerca dos entraves no uso do serviço de saúde pelos indivíduos quilombolas com diagnóstico autorreferido de HAS e assim ponderar que esta população segue invisibilizada pelos gestores que mantém uma lógica verticalizada na disponibilização de atendimento de saúde nos quilombos.

Nesse sentido, é preciso elucidar que não se trata de uma subutilização, porque o serviço não se encontra organizado para atender essa população em seus territórios, ou seja, tem sido os quilombolas que se adequam às barreiras do acesso e acessibilidade e vão em busca de atendimento mediante suas necessidades ditas emergenciais e não de promoção da saúde como preconiza a Política Nacional de Promoção da Saúde e o Serviço de Controle de hipertensão e diabetes.

AGRADECIMENTOS: Agradecemos às Associações de Moradores das Comunidades Quilombolas que aceitaram participar do estudo e colaboraram com a logística local das coletas.

CONTRIBUIÇÕES DOS AUTORES: Rosilene das Neves Pereira: concepção e desenho, da análise e interpretação dos dados, da redação do artigo. Ricardo Franklin de Freitas Mussi: concepção e desenho, da análise e interpretação dos dados, da redação do artigo. Claudio Bispo de Almeida: concepção e desenho, da análise e interpretação dos dados, da redação do artigo. Roseanne Montargil Rocha: revisão crítica de conteúdo intelectual importante. Todas(os) as(os) autoras(es) leram e aprovaram a versão final do manuscrito.

CONFLITOS DE INTERESSE: As(os) autoras(es) declaram que não há conflitos de interesse.

\section{REFERÊNCIAS}

Almeida, C.B., Santos, A.S., Vilela, A.B.A., \& Casotti, C.A. (2019). Reflexión sobre el control del acceso de quilombolas a la salud pública brasileña. Av. Enferm, 37(1), 92-103.

Andrade, S.S,A., Stopa, S.R., Brito, A.S., Cueri, O.S., Szwarcwald, C.L., \& Malta, D.C. (2015). Prevalência de hipertensão arterial autorreferida na população brasileira: análise da Pesquisa Nacional de Saúde, 2013. Epidemiol serv saúde, 24(2), 297-304.

Assis, L.C., Simões, M.O.S., \& Cavalcanti, A.L. (2012). Políticas públicas para monitoramento de hipertensos e diabéticos na atenção básica, Brasil. Rev bras pesqui saúde,14(2), 65-70.

Barros, S.M., Melo, A.P.S., \& Guimarães, M.D.C. (2014). Depressão em comunidades quilombolas no Brasil: triagem e fatores associados. Rev panam salud publica, 35(4), 256-263.

Belfort, I.K.P., Fernandes, M.A., Nunes, J.D.C., \& Monteiro, S.C.M. Elevação de níveis pressóricos em uma Comunidade Quilombola. Rev bras promoç saúde, 30(3), 1-8.

Bezerra, V.M., Andrade, A.C.S., Cesar, C,C., \& Caiaffa, W.T. (2013). Comunidades quilombolas de Vitória da Conquista, Bahia, Brasil: hipertensão arterial e fatores associados. Cad saúde pública, 29(9), 1889-1902. 
Bezerra, V.M., Andrade, A.C.S., César, C.C., \& Caiaffa, W.T. (2015). Desconhecimento da hipertensão arterial e seus determinantes em quilombolas do sudoeste da Bahia, Brasil. Cien saude colet, 20(3), 797-807.

Bezerra, V.M., Medeiros, D.S., Gomes, K.O., Souza, R., Giatti, L., Steffens, A.P., \& et al. (2014). Inquérito de Saúde em Comunidades Quilombolas de Vitória da Conquista/BA (Projeto COMQUISTA): aspectos metodológicos e análise descritiva. Cien saude colet, 19(6), 1835-1847.

Borges, D.B., \& Lacerda, J.T. (2018). Actions aimed at the Diabetes Mellitus control in Primary Health Care: a proposal of evaluative model. Saúde debate, 42(116), 162-178.

Borja, M.E.L., \& Pereira, C.D. (2018). As leis no 10.639/03 e no 11.645/08: reflexões a partir do pensamento crítico acerca da colonialidade do saber. Revista Cenas Educacionais, 1(1), 242-270.

Brasil. Ministério da Cultura. Fundação Cultural Palmares. (2016). http://www.palmares.gov.br

BRASIL. Ministério da Saúde. (2012). Secretaria de Vigilância em Saúde/Secretaria de Gestão Estratégica e Participativa, Ministério da Saúde. Vigitel Brasil 2011: vigilância de fatores de risco e proteção para doenças crônicas por inquérito telefônico. Brasília: Ministério da Saúde.

Carvalho Filha, F.S.S, Nogueira, L.T., \& Medina, M.G. (2014). Avaliação do controle de hipertensão e diabetes na Atenção Básica: perspectiva de profissionais e usuários. Saúde debate, 38(spe.), 265-278.

Chobanian, A.V., Bakris, G.L., Black, H.R., Cushman, W.C., Green, L.A., Izzo, J.L.,\& et al. (2003). The seventh report of the Joint National Committee on Prevention, Detection, Evaluation, and Treatment of High Blood Pressure: the JNC 7 report. JAMA, 289, (19), 2560-2572.

Fernandes, S.L. \&, Santos, A.O. (2019). Itinerários Terapêuticos e Formas de Cuidado em um Quilombo do Agreste Alagoano. Psicol cienc prof, 39(spe):e222592.

Ferreira, R.A., Barreto, S.M., \& Giatti, L. (2014). Hipertensão arterial referida e utilização de medicamentos de uso contínuo no Brasil: um estudo de base populacional. Cad saúde pública, 30(4), 815-826.

Ferreira, S.R.G., Moura, E.C., Malta, D.C., \& Sarno, F. (2006). Frequência de hipertensão arterial e fatores associados: Brasil, 2006. Rev saude publica, 43(2), 98-106.

Gerab, R.C., Ribeiro, A.S., Felicíssimo, A., \& Ramos, L.R. (2012). Controle de diabetes e hipertensão arterial na atenção primária à saúde em uma região do município de São Paulo. Rev adm saúde 14(57).

Gontijo, M.F., Ribeiro, A.Q., Klein, C.H., Rozenfeld, S., \& Acúrcio, F.A. (2012). Uso de anti-hipertensivos e antidiabéticos por idosos: inquérito de Belo Horizonte, Minas Gerais, Brasil. Cad saúde pública, 28(7), 1337-1346.

Kochergin, C.N., Proietti, F.A., \& César, C.C. (2014). Comunidades quilombolas de Vitória da Conquista, Bahia, Brasil: autoavaliação de saúde e fatores associados. Cad saúde pública, 30(7), 1487-1501.

Kurian, A.K., \& Cardarelli, K.M. (2007). Racial and ethnic differences in cardiovascular disease risk factors: a systematic review. Ethn Dis, 17(1), 143-152.

Lessa, I., Magalhães, L.; Araújo, M.J., Almeida, F.N., Aquino, E., \& Oliveira, M.M.C. (2006). Hipertensão arterial na população adulta de Salvador (BA) - Brasil. Arq bras cardiol, 87(6), 747-756.

Matos, F. B. de, Vasconcelos, L. R. C., Rocha, S. V., \& Mussi, R. F. de F. (2021). Subdiagnóstico da Hipertensão Arterial em adultos quilombolas de região baiana, Brasil. Research, Society and Development, 10(2), e37210211055.

Matta, S.R., Bertoldi, A.D., Emmerick, I.C., Fontanella, A.T., Costa, K.S., Luiza, V.L., \& et al. (2018). Fontes de obtenção de medicamentos por pacientes diagnosticados com doenças crônicas, usuários do Sistema Único de Saúde. Cad. Saúde Pública 34(3), e00073817.

Melo, M.F.T., \& Silva, H.P. (2015). Doenças crônicas e os determinantes sociais da saúde em comunidades quilombolas do Pará, Amazônia, Brasil. Revista da ABPN, 7(16), 168-189.

Mirando, R.D., Perrotti, T.C., Bellinazzi, V.R., Nóbrega, T.M., Cendoroglo, M.S., \& Neto, J.T. (2002). Hipertensão arterial no idoso: peculiaridades na fisiopatologia, no diagnóstico e no tratamento. Rev bras hipertens, 9(3), 293300.

Munger, M.A., Tassel, B.W., \& Lafleur, J. (2007). Medication nonadherence: an unrecognized cardiovascular risk factor. Med gen med, 19(9), 58.

Mussi, R.F.F., \& Petroski, E.L. (2019). Síndrome metabólica e fatores associados em quilombolas baianos, Brasil. Ciência \& Saúde Coletiva, 24(7), 2481-2490. 
Nogueira, D., Faerstein, E., Coeli, C.M., Chor, D., Lopes, C.S., \& Werneck, G.L. (2010). Reconhecimento, tratamento e controle da hipertensão arterial: estudo Pró Saúde, Brasil. Rev panam salud publica, 27(2), 103-109.

Novello, M.F., Rosa, M.L.G, Ferrerira, R.T., Nunes, I.G., Jorge, A.J.L., Correira, D.M.S., \& et al. (2017). Compliance with the Prescription of Antihypertensive Medications and Blood Pressure Control in Primary Care. Arq bras cardiol, 108(2), 135-142.

Paranhos, M. C. R., \& Cardoso, L. R. (2021). Body and health in the training of health professionals: a mapping of scientific production. Journal of Research and Knowledge Spreading, 2(1), e12480.

Pereira, R.N., Andrade, L.M., \& Amorin, D.S. (2018). O Serviço de Controle da Hipertensão e Diabetes como ferramenta para prevenção da doença renal crônica na ótica dos Profissionais da Atenção Básica. In: Convención Internacional de Salud, Cuba Salud, 2018, Cuba. Anais da III Convenção de Saúde Pública. Havana - Cuba, MS, 236-4705.

Pereira, R. das N., \& Mussi, R. F. de F. (2020). Acesso e utilização dos serviços de saúde da população negra quilombola: uma análise bibliográfica. ODEERE, 5(10), 280-303.

Pereira, R.N., Mussi, R.F.F., \& Rocha, R.M. (2020). Acesso e utilização dos serviços de saúde por quilombolas contemporâneos baianos. Revista da Associação Brasileira de Pesquisadores/as Negros/as (ABPN), 12(31).

Rosário, T.M., Scala, L.C.N., França, G.V.A., Pereira, M.R.G., \& Jardim, P.C.B.V. (2009). Prevalência, controle e tratamento da hipertensão arterial sistêmica em Nobres - MT. Arq bras cardiol, 93(6), 672-678.

Santos, A.T., \& Lucini, M. (2021). Educational practices and knowledge constituted in the political training processes of black activists: a literature review. Journal of Research and Knowledge Spreading, 2(1), e12356.

Secretaria de Políticas de Promoção da Igualdade Racial da Presidência da República (SEPPIR). (2010). Comunidades Quilombolas Brasileiras - Regularização Fundiária e Políticas Públicas. Brasília.

Silva, M.J.G., Lima, F.S.S., \& Hamann, E.M. (2010). Uso dos Serviços Públicos de Saúde para DST/ HIV/aids por Comunidades Remanescentes de Quilombos no Brasil. Saúde soc, 19(sup.2), 109-120.

Silva, R.A., \& Menezes, L.A. (2016). Os significados do uso de álcool entre jovens quilombolas. Rev latinoam cienc soc niñez juv, 14(1), 493-504.

Sociedade Brasileira de Hipertensão (SBH); Sociedade Brasileira de Nefrologia (SBN). (2010). VI diretrizes brasileiras de hipertensão. Arq bras cardiol, 95(sup.1):1-51.

Sturmer, G., Dias-da-Costa, J.S., Olinto, M.T.A., Menezes, A.M.B., Gigante, D.P., \& Macedo, S. (2006). 0 manejo não medicamentoso da hipertensão arterial sistêmica no Sul do Brasil. Cad saude publica, 22(8), 1727-1737.

Trad, L.A.B., Castellano, M.E.P., \& Guimarães, M.C.S. (2012). Acessibilidade à atenção básica a famílias negras em bairro popular de Salvador, Brasil. Rev saúde pública, 46(6), 1007-1013.

Travassos, C., \& Martins M. (2004). Uma revisão sobre os conceitos de acesso e utilização de serviços de saúde. Cad saúde pública, 20(sup.2), S190-S198.

Travassos, C., Oliveira, E.X, \& Viacava, F. (2006). Desigualdades geográficas e sociais no acesso aos serviços de saúde no Brasil: 1998 e 2003. Ciênc saúde coletiva, 11(4), 975-986.

Travassos, C., \& Viacava, F. (2007). Acesso e uso de serviços de saúde em idosos residentes em áreas rurais, Brasil, 1998 e 2003. Cad saúde pública, 23(10), 2490-2502.

Veronez, L.L., \& Simões, M.J.S. (2008). Análise da prescrição de medicamentos de pacientes hipertensos atendidos pelo SUS da rede municipal de saúde de Rincão - SP. Rev ciênc farm básica apl, 29(1), 45-51.

Viacava, F., Porto, S.M., Carvalho, C.C., \& Bellido, J.G. (2018). Desigualdades regionais e sociais em saúde segundo inquéritos domiciliares (Brasil, 1998-2013). Cien Saude Colet,

http://www.cienciaesaudecoletiva.com.br/artigos/desigualdades-regionais-e-sociais-em-saude-segundoinqueritos-domiciliares-brasil-19982013/16535.

World Health Organization (WHO). (2011). Non-communicable diseases country profile, 2011. http://www.who. int/nmh/publications/ncd_profiles2011/en/in dex.html

Recebido: 6 de maio de 2021 | Aceito: 22 de setembro de 2021 | Publicado: 04 de dezembro de 2021 